\title{
A HYBRID STOCHASTIC MODEL FOR CHARACTERIZATION OF SUBSURFACE HETEROGENEITY
}

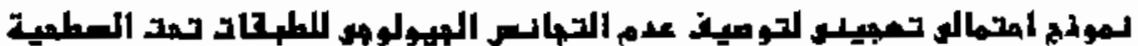

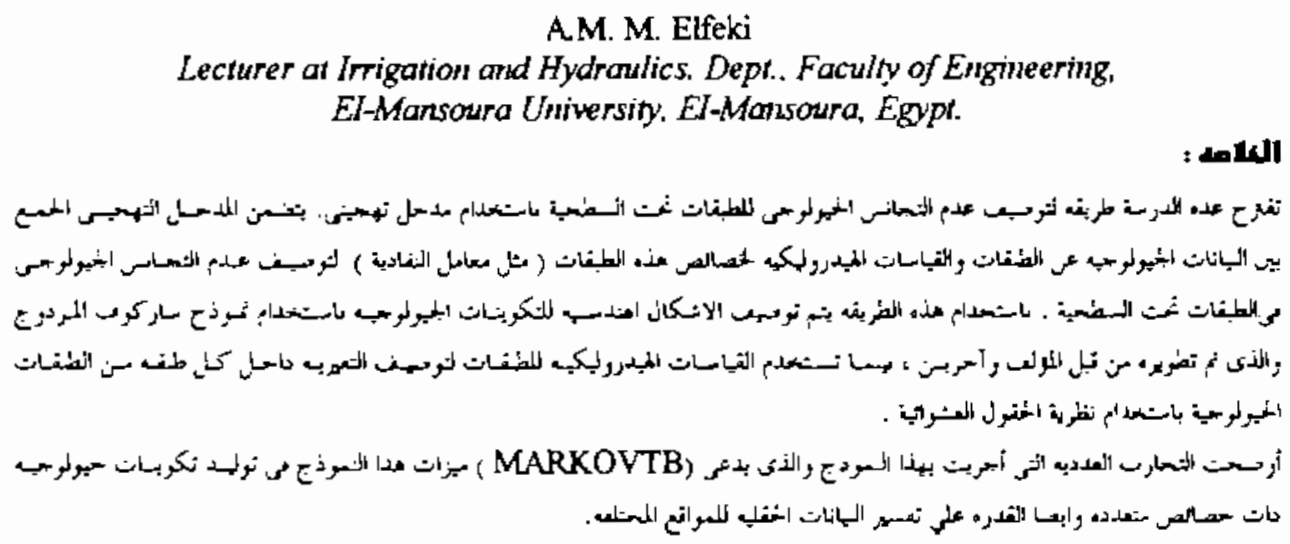

\section{ABSTRACT}

This study proposes a methodology for characterization of subsurface heterogeneity using a hybrid approach. The hybrid approach will incorporate soft geological information in addition to the available hard data to characterise geological heterogeneity under two scales of variability. The geometric shapes of the geologicai features are modelied stochastically based on soft information using the Coupled Markov Model developed by Elfeki et al. (1995), while hard data are used to model the variability inside these geometric shapes. This will lead to an improved characterization of the subsurface heterogeneity. The proposed hybrid model can be uscd to generate realizations of conductivity within the geological structure, with all realizations satisfying the "soft" geological knowledge and "hard" data.

\section{INTRODUCTrON}

Long time scale predictions by flow and transport models rely strongly on the appropriate description of the spatial structures of subsurface formations at various scales. These predictions are of utmost imponance in connection with groundwater resource management. Dcrailed knowledge of the subsurface variability is never available in a deterministic sense. An aiternative is stochastic modelling. A problem in stochastic methods is that the actual formation is often modelled by unimodal stationary random fields and, as a consequence, much of the actual spatial structure is lost. Moreover, stationary frelds are unrealistic in many geological settings. In practice, the natural variability observed in geological formations is nested, i.e., a geomctric structure is most often marifested with other internal fluctuations superimposed over the strucrure (e.g. Journel, ct al., 1978). This practical situation is not addressed in the geohydrological literature. From practical and economical point of view, it may not be feasible to fully characterise formation heterogeneity using hard data. Sone authors (Greihar, 1986; Journel, 1978) have suggested to incorporate subjective or soft data. This study is an attempt to integrate subjective geological information with measurements of hydraulic parameters (hard data).

Accepted September, 13,1997 


\section{STAGES OF THE HYBRID MODEL}

\section{Geometric Modelling of Large Scale Variability}

The purpose of this model is to identify geological features with discrete nature. The coupled Markov model is useci to characterise this scate of variebility. The geological information is coded in terms of transition probabilities to feed the computer programme "GEOSIM1" developed by the author. For illustration of the model reference is made to Elfeki (1996).

\section{Geostatistical Modelling of Local Scale Variability}

The tuming bands method applied by Tompson, et al., (1989) is used in this study to generate stationary fields of hydraulic conductivity within each geological unit. The log-conductivity field inside each unit is assumed to be independent from any other unit and it is modelled as

$Y(\mathbf{x})=\langle Y(\mathbf{x})\rangle+Y^{\prime}(\mathbf{x})$

where, $\mathbf{x}=(x, y)$ is a position inside a lithological unit, $Y(\mathbf{x})$ is log-conductivity at position $\mathbf{x}$,

$\langle Y(\mathbf{x})\rangle$ is the expected value of $\log$-conductivity at position $\mathbf{x}$ and $Y^{\prime}(\mathbf{x})$ is the stationary Gaussian field wich an expected value equal to zero and a variance equal to $\sigma^{2} Y(x)$. The field of $Y(\mathbf{x})=\log (K(\mathbf{x}))$ in the various units is transformed into a field of $K(\mathbf{x})$ by :

$X(\mathbf{x})=\exp [Y(\mathbf{x})]$

The spatial structure of the log-conductivity field in each geological unit is specified by an anisotropic exponential autocovariance function.

\section{IMPLEMENTATION OF THE HYBRID MODEL}

\section{Generation of The Geological Structure(Megascale Heterogeneity)}

The geological structure is sampled over intervals of a coarse grid. Transition probabilities between the different lithologies are determined and used for the simulation. A brief description of the steps are given here. The left and top boundaries are generated by the horizontal and vertical chains respectively in the same way as described by Elfeki (1996) At each cell location in the domain, the conditional probebilities of the coupled chains are calculated for all the states. A curnulative distribution of transition probabilities from the coupled chains is built. Monte-Carlo sampling to realise the states of the cell given the states of the neighbours is applied. The procedure is repeated until all cells of the simulation grid are assigned a state.

\section{Generation of Local Variability (Maeroscopic Heterogeneity)}

A finer grid is superimposed over the geological structure and each cell in the grid is coded with the prescribed lithology. A search algorithm is followed for assignment of values of hydraulic conductivity to each geological unit in the system. The following steps are considered. If $\mathrm{s}$ is positioned in lithology $S_{k}$, then $Y(x)=\left\langle Y_{k}\right\rangle+Y_{k}{ }_{k}, k=1, \ldots, n$, where $Y_{k}{ }_{k}$ is a statistically homogeneous Gaussian random field, generated by the tuming bands algorithm. 
The search algorithm is repeated to cover all the cells in the dornain and each ceil is assigned a value corresponds to parameters of its statistical distribution and its statistical anisotropy pattern. Then the conductivity value is catculated by the anti-logarithm as, $K(\mathbf{x})=\exp (Y(\mathbf{x}))$.

\section{ILLUSTRATYON OF THE MODEL RESULTS BY SOME SYNTHETTC EXAMPLES}

A set of non-starionary two-dimensional fields is generated by the models "GEOSTM1" and "MARKOVTB".developed by the author. The model is illustrated in Fig. 1. Here, a 2D vertical cross-section of large scale geological structure is shown with dimensions of $2000 \mathrm{~m}$ in length and $400 \mathrm{~m}$ in depth (see top of Fig 1 ). It is assurned that the geological system consists of four lithological units coded 1, 2, 3 and 4 in the figure and indicated by colour white, light grey, dark grey and black respectively. The stricture is generated by the Markov method with transition probabilities given in Table 1, sampled over incervals of $20 \mathrm{~m}$ in the horizontal direction and $10 \mathrm{~m}$ in the vertical direction. A finer grid with cell dimensions of $10 \times 5 \mathrm{~m}$ is superimposed over the structure for the generation of conducrivity field. The conductivity variations inside each geological unit is generated by the computer model "MARKOVTB" with various statistics in order to investigate various cases of non-stationary fields. Parameters of each case are displayed in tables $2,3,4,5$ respectively The sirnulation results of these cases will be discussed in the following sections

Table 1 Statistics used to generare the geological structure in Fig 1.

Length of the given section $(m)=2000, \quad$ Depth of the given section $(m)=400$

Sampling interval in X-axis $(m)=20 \quad$ Sampling interval in $Y$-axis $(m)=10$.

Horizontal Transition Probability Matrix

\begin{tabular}{lcccccccc} 
& \multicolumn{3}{c}{ Input Statistics to the Model } & \multicolumn{4}{c}{ Calculated Statistics from Fig. 1a } \\
State & 1 & 2 & 3 & 4 & 1 & 2 & 3 & 4 \\
1 & 0.960 & 0.010 & 0.010 & 0.020 & 0.933 & 0.007 & 0.021 & 0.039 \\
2 & 0.010 & 0.970 & 0.010 & 0.010 & 0.015 & 0.964 & 0.012 & 0.009 \\
3 & 0.010 & 0.020 & 0.960 & 0.010 & 0.006 & 0.026 & 0.957 & 0.011 \\
4 & 0.010 & 0.010 & 0010 & 0.970 & 0.016 & 0.002 & 0.014 & 0.968
\end{tabular}

Vertical Transition Probability Matrix

Input Statistics to the Model Calculated Statistics from Fig. la

$\begin{array}{lcccccccc}\text { Stare } & 1 & 2 & 3 & 4 & 1 & 2 & 3 & 4 \\ 1 & 0.930 & 0.010 & 0.030 & 0.030 & 0.810 & 0.041 & 0.053 & 0.096 \\ 2 & 0.100 & 0.800 & 0.050 & 0.050 & 0.044 & 0876 & 0.071 & 0.010 \\ 3 & 0.050 & 0.050 & 0800 & 0.100 & 0.042 & 0.068 & 0.805 & 0.086 \\ 4 & 0.020 & 0.050 & 0.040 & 0.870 & 0.019 & 0.077 & 0.058 & 0.846\end{array}$

\section{DISCUSSION OF THE MODEL RESULTS}

\section{Case (a): Non-Stationarity in The Mean}

It is assumed that each geological unit possesses a different mean conducrivity, while the variance and correlation lengths are assumed equal. The simulation parameters used are displayed in Table 2. Fig. Za shows some conductivity profiles. In Fig. la it can be seen that the boundarics between the geological units are still clear and not smeared by the influence of the local variability, in this case due to the large contrast in the mean. 
Table 2 Simulation Parameters (Non-Stationarity in The Mean).

\begin{tabular}{|c|c|c|c|c|c|c|c|}
\hline $\begin{array}{l}\text { Parameter } \\
\text { State }\end{array}$ & $w_{i}$ & $\begin{array}{c}\langle K\rangle \\
\mathrm{m} / \mathrm{day}\end{array}$ & $\begin{array}{c}\sigma K \\
\mathrm{~m} / \mathrm{day}\end{array}$ & $\langle Y\rangle$ & $\sigma \gamma$ & $\begin{array}{c}\lambda_{x} \\
(\mathrm{~m})\end{array}$ & $\begin{array}{c}\lambda_{v} \\
(\mathrm{~m})\end{array}$ \\
\hline 1 & 0.13 & 100. & 10. & 4.6 & 0.10 & 200. & 50 \\
\hline 2 & 0.31 & 50. & 10. & 3.9 & 0.20 & 200. & 50. \\
\hline 3 & 0.31 & 10. & 10. & 1.96 & 0.83 & 200. & 50. \\
\hline 4 & 0.25 & 1. & 10. & -2.3 & 2.15 & 200. & 50. \\
\hline
\end{tabular}

where, $w_{i}$ is the marginal probability of state $i,\langle K\rangle$ is the mean conductivity of state $i, \sigma_{K}$ is the standard deviation of state $i,\langle Y\rangle$ is the logarithrnic transform of $\langle K\rangle$, $\sigma \gamma$ is the logtransform of $\sigma_{K}, \lambda_{x}$ and $\lambda_{y}$ are the correlation lengths of each state in $\mathrm{x}$ and $\mathrm{y}$ directions respectively.

\section{Case (b): Non-Stationarity in The Variance}

It is assumed that the geological units possess different conductivity variances, while the arithmetic mean and the correiation lengths are constant. The simulation parameters used are displayed in Table 3. Fig. $2 \mathrm{~b}$ slows a conductivity profile In Fig. $1 \mathrm{~b}$ it can be seen that the structurc is smeared by the variability.

Table 3 Simulation Parameters (Non-Stationarity in The Variance).

\begin{tabular}{|l|c|c|c|c|c|c|c|}
\hline $\begin{array}{l}\text { Parameter } \\
\text { State }\end{array}$ & $w_{i}$ & $\begin{array}{c}\langle K\rangle \\
\text { m/day }\end{array}$ & $\begin{array}{c}\sigma_{K} \\
\text { m/day }\end{array}$ & $\langle\eta$ & $\sigma Y$ & $\begin{array}{c}\lambda_{x} \\
(\mathrm{~m})\end{array}$ & $\begin{array}{c}\lambda_{V} \\
(\mathrm{~m})\end{array}$ \\
\hline $\mathrm{I}$ & 0.13 & 5. & 20. & 0.19 & 1.68 & 100 & 10. \\
\hline 2 & 0.31 & 5 & 10. & 0.80 & 1.27 & 100 & 10. \\
\hline 3 & 0.31 & 5. & 5. & 1.26 & 0.83 & 100 & 10 \\
\hline 4 & 0.25 & 5 & 2. & 1.54 & 0.39 & 100. & 10. \\
\hline
\end{tabular}

\section{Case (c): Non-Stationarity in The Correlation Lengths}

It is assumed that the geological units possess different correlation lengths but the arithmetic mean of conductivity, the variance and the shape of the auto-covariance function are equal. The parameters used are displayed in Table 4 . Fig. Ic shows the conductivity field and Fig.2c shows a conductivity profile. In these figures it can be seen that the boundaries are smeared, but from the local variability one can notice diffcrent correlation patterns in the generated field.

Table 4 Simulation Parameters (Non-Stationarity in Correlation Lengths).

\begin{tabular}{|l|c|c|c|c|c|c|c|}
\hline $\begin{array}{l}\text { Parameter } \\
\text { State }\end{array}$ & $w_{i}$ & $\begin{array}{c}\langle(\lambda) \\
\mathrm{m} / \text { day }\end{array}$ & $\begin{array}{c}\sigma K \\
\text { m/day }\end{array}$ & $\langle(\rangle)$ & $\sigma_{Y}$ & $\begin{array}{c}\lambda_{x} \\
(\mathrm{~m})\end{array}$ & $\begin{array}{c}\lambda_{\nu} \\
(\mathrm{m})\end{array}$ \\
\hline 1 & 0.13 & 10. & 5. & 2.19 & 0.47 & 50. & 50. \\
\hline 2 & 0.31 & 10. & 5. & 2.19 & 0.47 & 100. & 50. \\
\hline 3 & 0.31 & 10 & 5. & 2.19 & 0.47 & 200. & 10. \\
\hline 4 & 0.25 & 10 & 5. & 2.19 & 0.47 & 500. & 5. \\
\hline
\end{tabular}




\section{Case (d): Globalty Non-Stationarity}

It is assumed that the geological units possess different parameters: arithmetic mean of hydrautic conductivity, variance and correlation lengths, whilc the auto-covariance functions are equal. This case represents a real field situation. The simulation parameters used are displayed in Table 5. Fig.ld shows the conductivity held generated and Fig.2d shows a conductivity profile. In these figures it can be seen that the boundaries are remarkabie like case (a). The non-stationarity in the mean is therefore the major reason of having pronounced boundaries.

Table 5 Simulation Parameters (Globally Non-Stationarity).

\begin{tabular}{|c|c|c|c|c|c|c|c|}
\hline $\begin{array}{l}\text { Parameter } \\
\text { State }\end{array}$ & $\overline{w_{i}}$ & $\begin{array}{r}\langle\boldsymbol{X}\rangle \\
\mathrm{m} / \mathrm{day}\end{array}$ & $\begin{array}{c}\sigma_{K} \\
\mathrm{~m} / \text { day }\end{array}$ & $\langle h\rangle$ & $\sigma y^{\prime}$ & $\begin{array}{l}\lambda_{x} \\
\text { (m) }\end{array}$ & $\begin{array}{l}\lambda_{v} \\
(\mathrm{~m})\end{array}$ \\
\hline 1 & 0.13 & 50 & 50. & 357 & 0.83 & 50. & 50. \\
\hline 2 & 0.31 & 10. & $\overline{5}$. & 2.19 & 0.47 & 100. & 50. \\
\hline 3 & 0.31 & 1.0 & 2. & -0.80 & 1.0 & 200. & 20 \\
\hline 4 & 0.25 & 0.1 & 0.5 & -3.93 & 1.81 & 500. & 5 \\
\hline
\end{tabular}

\section{Interpretation of The Model Results by Variogram Analysis}

Variograms of $\log (\boldsymbol{K})$ have been estimaled in $x$ direction for all previous cases in order to interpret the influence of various types of non-stationarity on the global variogram behaviour. This may help in analysing variograms of real field sicuation. Fig. 4 displays variograms of all cases mentioned earlier, in $x$ direction. For ail the cases mentioned above, equivalent globally stationary fields are generated to evaluate the validity of the global-stationarity assumption. The fields are presented in Fig.3. The variograms of the fields are calculated and plotted in Fig. 4 for comparisons. Table 6 displays the statistical parameters used to generate the equivalent stationary fields.

Table 6 Simulation Parameters for Equivalent Global Stationary Fields.

\begin{tabular}{||c|c|c|c|c|c|c|}
\hline $\begin{array}{l}\text { Parameter } \\
\text { Case }\end{array}$ & $\begin{array}{c}\left\langle K_{m}\right\rangle \\
\mathrm{m} / \text { day }\end{array}$ & $\begin{array}{c}\sigma_{m} \\
\mathrm{~m} / \text { day }\end{array}$ & $\left\langle Y_{m}\right\rangle$ & $\sigma_{m} \gamma^{\prime}$ & $\begin{array}{l}\left\langle\lambda_{x}\right\rangle \\
(\mathrm{m})\end{array}$ & $\begin{array}{l}\left\langle\lambda_{\nu}\right\rangle \\
(\mathrm{m})\end{array}$ \\
\hline $\mathrm{a}$ & 31.85 & 34.5 & 3.07 & 0.881 & 200. & 50. \\
\hline $\mathrm{b}$ & 5 & 10.8 & 0.74 & 1.32 & 100 & 10. \\
\hline $\mathrm{c}$ & 10 & 5 & 2.19 & 0.47 & 224.5 & 26 \\
\hline $\mathrm{d}$ & 9.935 & 263 & 1.26 & 1.44 & 224.5 & 29.5 \\
\hline
\end{tabular}

where, $\left\langle K_{m}\right\rangle$ is the global mean of thc statcs, $\sigma_{m}$ is the global standard deviation of the states. $\left\langle Y_{m}\right\rangle, \sigma_{m} Y$ are the corresponding $\log$-transforms, and $\left\langle\lambda_{y}\right\rangle,\left\langle\lambda_{y}\right\rangle$ are the average correlation lengths of the states.

In case (a), non-stationarity in the mean, the non-stationarity in the variogram is most pronounced compared with the other cases, since the variogram does not seem to reach a constant value (sill) and it is growing with the spatial lag. From Fig.4a it is clear that the stationary variogram is far from the non-stationary variogram, which means that the assumption of globally stationarity field in this case is not applicable.

In case (b), non-stationarity in the variance, the non-stationarity behaviour is less pronounced. The reason for that is the influence of high variances and constant mean which smear the 
boundaries between the different geological units. This can be observed clearly in the profiles of Fig. 2 b. Fig. 4 b shows a reasonable agreement (practically) between variogram shapes in case of non-stationarity and globally stationary field. One can notice that the use of an equivalent globalty stationary field to represent the non-stationary is not proper although the variogram shapes look relatively close at further lags. This means that sometimes non-stationarity of the data cannot be observed clearly in the variogram.

In case (c), non-stationarity in the correlation lengths, the variogram looks quite stationary. This means that there is no significant influence of non-stationarity of the correlation parterns of the individual units on the global variogram behaviour. Estimation of the (asymptotic) sill variance from Fig. $4 \mathrm{c}$, which is about 0.22 is in perfect agreement with the variance from Table 4 that is $(0.47)^{2}=0.221$. This ensures good reproduction of the variance in the simulated field. Fig $4 \mathrm{c}$ shows a relatively fair agreement in the variorgan shapes in case of the nonstationary field and its equivalent globally stationary field. This indicates that non-stationarity in correlation patterns can not be observed in the variogram.

In case (d), globally non-stationarity, the variograms in Fig.4d is far apart, as similar to case (a). This can be attributed to the distincrion in the mean conductiviries of different geological units which are quite pronounced. The assumption of equivalent stationarity field does not hold, which means that serious errors can be introduced due to neglecting non-stationarity.

\section{CONCLUSIONS}

The following concluding rernarks are drawn from the study:

(1) Geological krowledge and expertise of subjective nature (translated into transition probabilities) can be combined with quantitative geostatistical models (in terms of conrelated randorn fields) to produce more accurate realizations of lithology and hydraulic conductivity fields of underground reservoirs.

(2) The proposed hybrid model can be used to generate realizations of conductivity within the geological structure, with all realizations satisfying the "soft" geological knowledge (reproduction of transition probabilities) and "hard" dara (reproduction of PDFs and its moments).

(3) From the synthetic examples, it has been shown that high contrast in the mean of individual populations is the main factor of pronounced non-stationarity in comparison with other types of non-stationarity.

(4) The assumption of a globally stationary random function model is not adequate to describe many fields as far as the non-stationarity in the mean is concemed.

(5) Unless the non-stationarity of the data is clearly pronounced, it can not be manifested by variogram analysis.

\section{REFERENCES}

1. ELFEKI, A. M. \& UFFINK, G. J. M. \& BARENDS, F. J. B. (1995). Stochastic simulation of heterogeneous geological formations using soft information, with an application to groundwater. In Groundwater Quality: Remediation and Protection, QG'95. Edited by Kovar, $\mathrm{K}$. and Krasny, IAHS Publication No. 225. 
2 ELFEKI. A.M. (1996) Stochastic characterization of geological heterogeneity and its impact on groundwater contaminant transport, $\mathrm{PhD}$. thesis at Delft University of Technology, The Netherlands.

3. GELHAR, L. W. (1986). Stochastic subsurface hydrology: from theory to appiications. Water Resources. Research. 22(9): 135S-145S.

4 JOURNEL, A. G. \& HUIJBREGTS, C. (1978) Mining geostacistics. New York: Academic Press, 600p.

5. TOMPSON, A. F B. \& ABABOU, R. \& GELHAR. L. W (1989) Impiementation of the three-dimensional turning bands random tield generator Water Rcsources Research. 25(10): 2227-2243

6. Christakos, G. (1992). Random Fields Models in Earth Sciences Acadcmic, San Diego, Catif

7 Haldorscn. H. H \& Damsleth. E (1990). Stochastic Modelting Society of Petroleum Engincers, SPE 2032 l. 


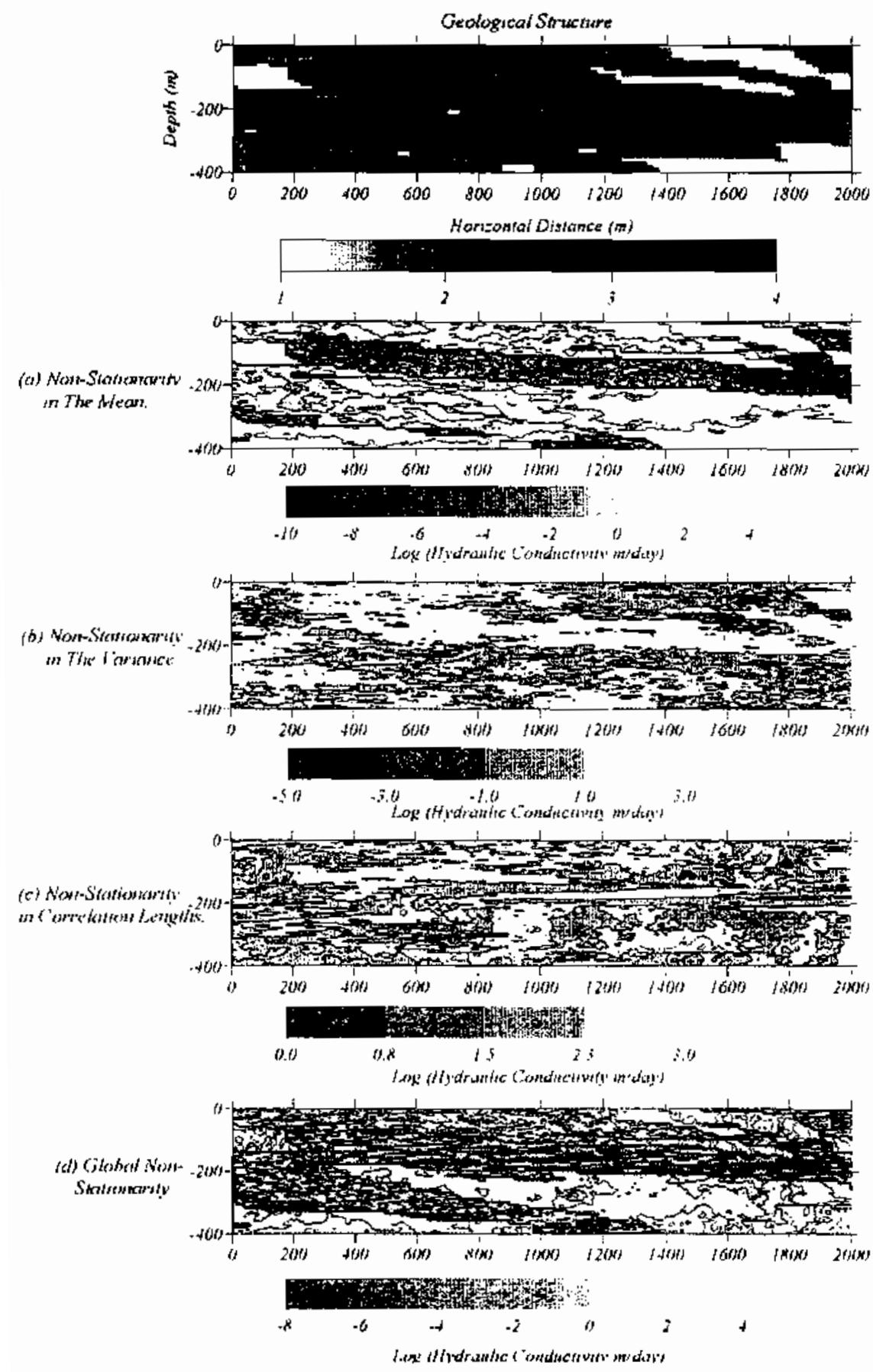

Fig.l Demonstration of The Hybrid Model with Some Synthetic Cases. 

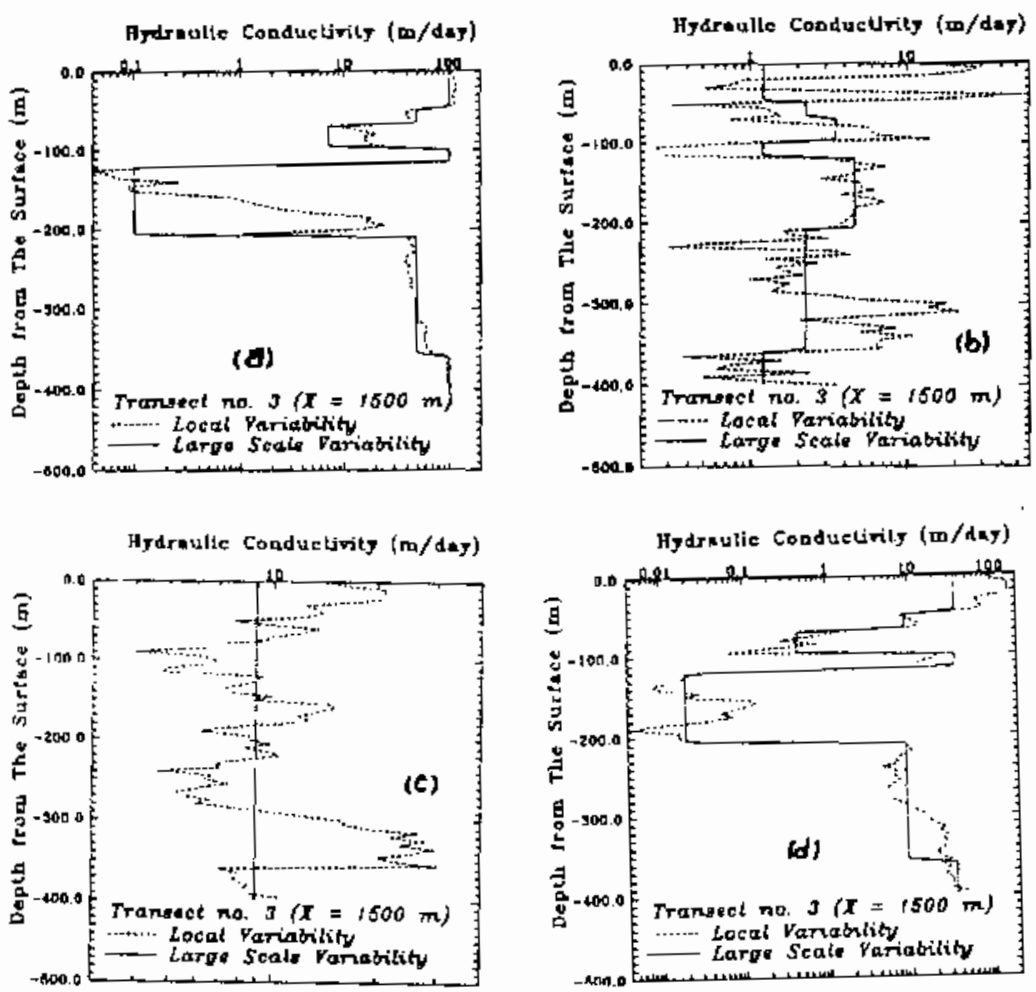

Fig. 2 Conductivity Profiles for All Cases (a) Nonstationarity in the Mean, (b) Nonstationarity in The Variance, (c) Nonstationarity in Correlation Lengths, (d) Global Nonstationarity. 


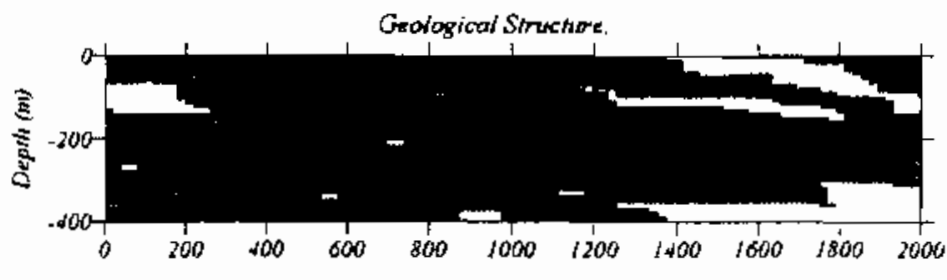

(a)

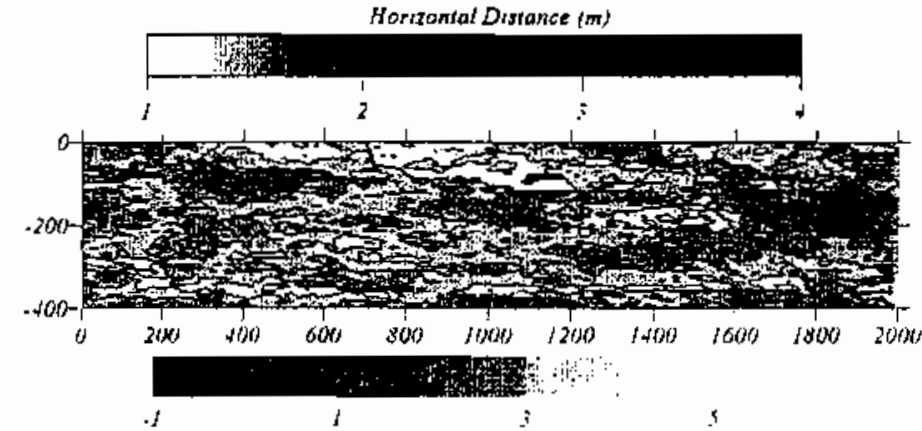

(b)

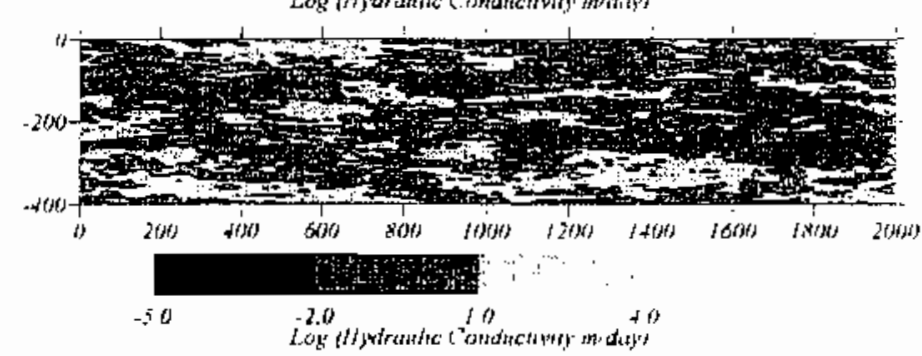

(c)

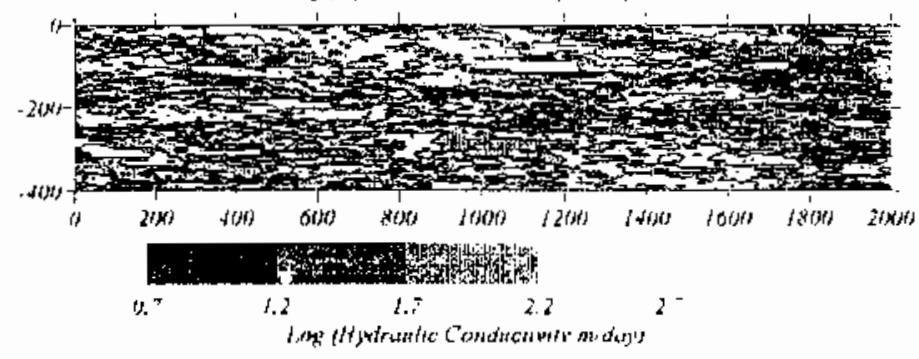

(d)

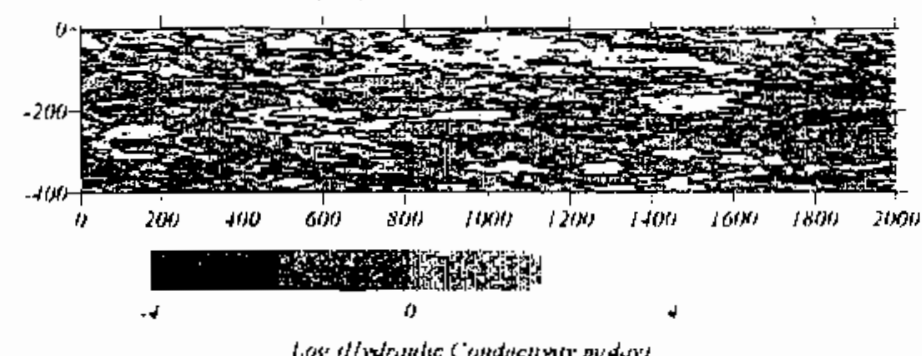

Fig.3 Equivalent Stationary Fields of The Synthetic Examples. 

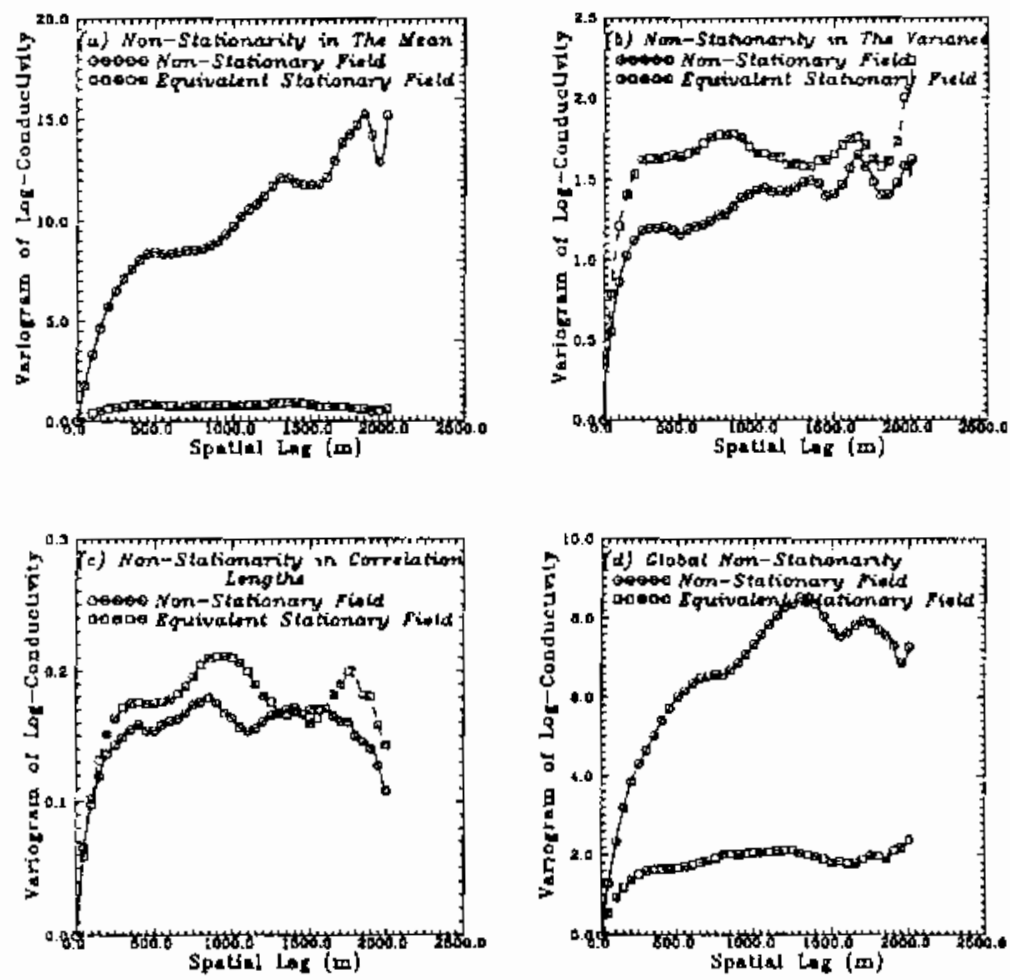

Fig.4 Variograms of Log-Conductivity for All Cases in X-direction. 